\title{
PREVALENCE OF HEPATITIS B VIRUS AMONG THE PHYSICIANS OF DEPARTMENT OF MEDICINE OF DHAKA MEDICAL COLLEGE HOSPITAL, DHAKA
}

\author{
SAHA JK ${ }^{1}$, AZAD KAK ${ }^{2}$, HOSSAIN MZ $^{3}$, AMIN MR ${ }^{4}$, WAZIB A ${ }^{5}$, RAHMAN MK ${ }^{6}$
}

\begin{abstract}
Introduction: Hepatitis B virus is encountered sporadically throughout the year in Bangladesh. Bangladeshi physicians are also in a great risk for this blood-borne virus. To find out the seroprevalence of HBV and vaccination rate among the physicians, was the aim of this study.

Methods: The study was an observational study and was conducted in the department of Medicine of Dhaka Medical College Hospital, Dhaka between April and September, 2012. Eighty physicians of department of Medicine were searched for hepatitis $B$ virus with the help of a structured questionnaire. Physicians, who were found not vaccinated, were also investigated for HbsAg positivity by ELISA method.

Results: Eighty five percent physicians were found vaccinated. Remaining 15\% (12/80) physicians were investigated for $\mathrm{HbsAg}$ positivity but all were found negative. Vaccinated physicians who checked antibody titre (35.3\%), were found well immunized. Fifty percent physicians experienced needle stick injury 2-5 times in their lifetime and 20\% physicians did not use protective equipments during any invasive procedure.

Conclusion: HBV remains a major cause of morbidity and mortality in Bangladesh. Hence, our physicians who were not vaccinated and also found HbsAg negative, should complete their vaccination schedule as early as possible.
\end{abstract}

Key Words: Hepatitis B virus (HBV), HBV vaccination, HbsAg, antibody titre, physicians.

J Dhaka Med Coll. 2013; 22(1) : 11-15.

\section{Introduction:}

Hepatitis B is a disease caused by the hepatitis $B$ virus (HBV), which is transmitted through percutaneous or mucosal exposure to infectious blood or body fluids ${ }^{1}$. It is a major problem because it can cause chronic infection, resulting in cirrhosis of the liver, liver cancer, liver failure, and death. In addition, several extra-hepatic lesions occur because of HBV infection, with this, there is deposition of immune complexes in different organs of the body especially, the kidney ${ }^{2}$. Persons with chronic infection also serve as the main reservoir for continued HBV transmission ${ }^{1}$.

HBV accounts for an estimated 360 million chronic infections ${ }^{3}$ with about a million who die each year from chronic liver diseases ${ }^{4}$. Most persons who become chronic carriers of the virus live in Asia and Africa ${ }^{5}$. These regions are said to be highly endemic for hepatitis B. In South Africa, over $50 \%$ of the population have been infected by the virus, and at least 3 million people are chronic HBV carriers ${ }^{4}$.

The major route of HBV transmission is horizontal (i.e. transmission unrelated to recognised sexual, perinatal, or parenteral exposure $)^{6}$ in children under 5 years of age; however, percutaneous/ parenteral transmission is also an important mode of $\operatorname{spread}^{7}$.

Health care workers (HCWs) like the physicians may be exposed to the risk of infection with blood-borne viruses (BBVs) such

1. Dr. Jayanto Kumar Saha, Medical Officer, Out-Patient Department, Dept. of Medicine, Dhaka Medical College Hospital, Dhaka.

2. Prof. Khan Abul Kalam Azad, Professor, Dept. of Medicine, Dhaka Medical College Hospital, Dhaka.

3. Dr. Mohammad Zaid Hossain, Assistant Professor, Department of Medicine, Dhaka Medical College, Dhaka.

4. Dr. Md. Robed Amin, Associate Professor, Dept. of Medicine, Dhaka Medical College Hospital, Dhaka.

5. Dr. Amit Wazib, MD student, Dhaka Medical College, Dhaka.

6. Dr. Kazi Monisur Rahman, Assistant Professor, Diabetic Association Medical College, Faridpur

Correspondence: Dr. Jayanto Kumar Saha, Medical Officer, Out-Patient Department, Dept. of Medicine, Dhaka Medical College Hospital, Dhaka. 
as $\mathrm{HBV}$, hepatitis $\mathrm{C}$ virus $(\mathrm{HCV})$ and human immunodeficiency virus (HIV) via contact with blood (and other body fluids) in the course of their work $^{8}$. The form of exposure most likely to result in occupational BBV infection is a needle stick injury (NSI). A study of $554 \mathrm{HCWs}$ conducted in Kenya to establish their immunization status, found that only $12.8 \%$ (71/554) of HCWs had received vaccination previously and none had been screened for immunity or hepatitis B surface antigen $(\mathrm{HBsAg})^{9}$. In this study $55 \%$ of $\mathrm{HCWs}$ were unprotected, thereby predisposing them to HBV infection. A study on South African doctors in Bloemfontein found that $81 \%$ had previously been vaccinated ${ }^{10}$.

HBV can be prevented by strict adherence to standard microbiological practices and techniques, and routine use of appropriate barrier precautions to prevent skin and mucous membrane exposure when handling blood and other body fluids of all patients in health care settings ${ }^{4}$. Following exposure to blood or body fluids, post-exposure prophylaxis can be administered as a combination of passive immunization with hepatitis B immunoglobulin (HBIG) and vaccination with the hepatitis $B$ vaccine ${ }^{4}$. However, the most cost-effective method to prevent and control hepatitis B is through pre-exposure vaccination ${ }^{4}$.

It is important for the physicians to know their HBV status by being screened for the HBV surface antigen (HBsAg) and antibody (anti$\mathrm{HBs}$ ), and to be vaccinated against hepatitis B if found to be unprotected. This will protect them from being infected, and prevent them from spreading the virus which can infect patients. The vaccine has been found to be safe and effective, and can protect one for a lifetime ${ }^{4}$. Education and prevention of infection with HBV should be emphasized, and all patients should be regarded as potential HBV carriers regardless of their medical history or condition.

\section{Methods:}

The study was an observational study and was conducted in the department of Medicine of Dhaka Medical College Hospital, Dhaka between April and September in 2012. Eighty physicians of department of Medicine were searched for hepatitis B virus with the help of a structured questionnaire. Physicians, who were found not vaccinated, were also investigated for $\mathrm{HbsAg}$ positivity by ELISA method.

\section{Inclusion criteria:}

1. Physicians who were willing to participate in the study,

2. Physicians who had been exposed to clinical setting.

\section{Exclusion criteria:}

1. Physicians who were not willing to participate,

2. Physicians who were not available during the period of data collection.

\section{Data collection:}

After selection of a physician, he was investigated according to a Questionaire. If he was found not vaccinated, he was screened for HbsAg positivity. If he was found positive, then he was further investigated whether he should get proper treatment. If someone was found HbsAg positive and also getting treatment for it, his data was also collected with greater emphasis. If a Physician was found not vaccinated and also HbsAg negative, he was advised for vaccination as early as possible. Vaccinated physicians were further enquired for their vaccination doses and antibody titre after vaccination. All the collected data were entered in the SPSS version 17.0.

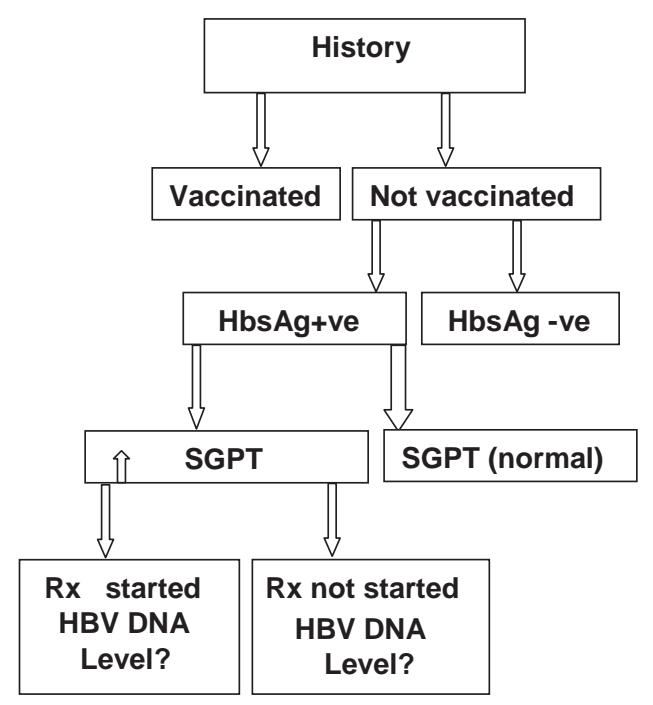




\section{Results:}

Eighty physicians of Medicine department of Dhaka Medical College Hospital were studied. Physicians' age ranged from 23 to 59 years. $77.5 \%$ of them were young (age 23-30 years). Most of them (56.70\%) were working for only less than one year. The total number of male physicians were 60 (75\%) and females were 20 (25\%). Out of 80 physicians, 68 (85\%) were found vaccinated. Twelve (15\%) physicians were not vaccinated and they were investigated and found HBsAg negative. Among the 68 vaccinated physicians, 24 physicians (35.3\%) checked their immune status after their vaccination and they all were found protected. However, still most of the vaccinated physicians $(44 / 68,64.7 \%)$ did not checked their antibody titre after vaccination.Among these vaccinated physicians, 41 of them were vaccinated properly with 3 doses of vaccine, 26 of them were vaccinated with 2 doses and one of them forgot his vaccination doses. Twenty four vaccinated physicians checked their vaccination status and they all were found protected. One physician with vaccination with two doses was also found well protected after checking his immune status. Among the 80 physicians, 50\% (40/80) of them experienced needle stick injury2-5 times in their lifetime, $12.5 \%(10 / 80)$ once, $12.5 \%(10 / 80) 6-10$ times, $10 \%(8 / 80)$ more than 10 times in their life time. Fifteen percent $(15 \%, 12 / 80)$ of them had no needle stick injury in their lifetime. $82.35 \%(56 / 68)$ of the physicians who had needle stick injury never report it to someone. One of the physicians received post exposure prophylaxis after needle stick injury. Among the physicians, 20\% (16/80) reported 2-5 times splashing of blood and body fluid in their eyes or open body surface, 5\% (4/80) more than 10 times, $10 \%$ (8/80) 6-10 times, 10\% (8/80) once in their lifetime and 55\% (44/80) had never experienced this. At least, 45\% (36/80) physicians experienced splashing of blood and body fluid in their open body surface once or more in their lifetime. Among these physicians, 30\% (24/80) always uses protective clothing and equipments, 50\% (40/80) sometimes and 20\% (16/80) never uses these while examining a patient. Seventy five percent of them consider HBV vaccine expensive. However, all of them strongly agree that $H B V$ vaccination should be compulsory for every physician.

\section{Discussion:}

According to the epidemiological study of Mahtab et al. ${ }^{11}$, prevalence of Hepatitis B virus in Bangladesh is 5.5\%. In our study, 85\% (68/ 80) physicians were found vaccinated and $15 \%$ $(12 / 80)$ physicians were not vaccinated but they all were HBsAg negative. Vaccinated physicians who checked their immune status, were all found to be protected. Among the 68 vaccinated physicians,24 physicians checked their immune status after their vaccination and they all were found protected.One physician was also found well protected who only received 2 doses of vaccination. But most of the vaccinated physicians(44/68,64.7\%) did not checked their antibody titre after vaccination. One physician received post exposure prophylaxis after needle stick injury. A South African study carried out in Bloemfontein, found that $81 \%$ of doctors had previously been vaccinated against hepatitis B infection ${ }^{10}$. A study in Dublin, Ireland, found that whilst the majority (83\%) of HCWs (health care workers) were vaccinated against HBV with the required 3 doses of hepatitis $b$ vaccine and also checked their immunity, this was influenced by the knowledge about the benefits given by the vaccine ${ }^{12}$. A good response (i.e. $\geq 10 \mathrm{mIU} / \mathrm{ml}$ ) will impart long-lasting immunity ${ }^{13}$. It is important to emphasize that adherence to licensed hepatitis $B$ vaccination schedules results in a protective concentration of anti-HBs that is $10 \mathrm{mIU} / \mathrm{ml}$ in $90-100 \%$ of healthy infants; children and adults ${ }^{14}$. In this study, 50\% (40/80) physicians experienced needle stick injury 2-5 times in their lifetime. A Birmingham, UK study demonstrated 53\% $(40 / 75)$ of doctors had experienced a $\mathrm{NSI}^{15}$. Needle stick injuries, especially those involving hollow needles, have been reported as the most common route of transmission (Alam, 2002; De Villiers et al, 2007) ${ }^{10}$. According to the study of Mahtab et al. ${ }^{11}$, major risk factors for exposure to $\mathrm{HBV}$ included treatment by non medical, "Quack" practitioners (85.71\%), intravenous injections (32.14\%) or 
infusions (71.43\%) and barbers (42.86\%). The importance of checking antibody levels after vaccination is illustrated by the South African study carried out in Bloemfontein, where it was found that $81 \%$ of doctors exposed to NSIs (needle stick injury) said they had previously been vaccinated against hepatitis B infection. Moreover, amongst HCWs (Health Care Workers) that were exposed to a NSI, there were two of them that seroconverted, and it was found that they had not been previously vaccinated. Seemingly, most assumed they were immune since only $21.7 \%$ underwent serological testing for HBV directly after the NSI ${ }^{10}$. In our study, $45 \%(36 / 80)$ physicians experienced splashing of blood and body fluid in their open body surface at least once or more in their lifetime. $30 \%$ of physicians always and $50 \%$ physicians sometimes used protective clothing and equipments while examining patient or during any invasive procedure, but $20 \%$ physicians never used these while examining patients. In Birmingham, UK study, more nurses $(86 \%)$ than doctors $(41 \%)$ had the attitude that all patients should be treated as if they have a blood-borne pathogen. The nurses also had statistically significantly better attitudes towards universal precautions in general (washing hands before and after dealing with patients, and wearing gloves when drawing blood) than doctors, which translated into better practices than doctors. Birmingham, UK study also demonstrated in hand washing where $58.7 \%$ and $64.3 \%$ of nurses always washing their hands before and after patient contact, compared to $10.7 \%$ and $26.7 \%$ of doctors. Similarly, $56.6 \%$ of nurses always wore gloves when taking blood, compared to $10.7 \%$ of doctors $^{15}$. In this study, $75 \%$ physicians considered HBV Vaccine expensive. But all of the physicians who participated in this study strongly agreed that HBV Vaccination should be compulsory for every physician. Physicians who were found not vaccinated yet, should complete their vaccination schedule as early as possible.

This study has also some limitations. Most of the vaccinated physicians did not checked their antibody titre. Among the 68 vaccinated physicians, 24 physicians (35.3\%) checked their immune status after their vaccination and they all were found protected.But still most of the vaccinated physicians $(44 / 68,64.7 \%)$ did not check their antibody titre after vaccination. So,it is not sure that whether they were really protected from this virus or not. Another thing is co-infection with $\mathrm{HCV}$ (hepatitis C virus) or HIV (human immune virus) was not searched in this study.

\section{Conclusion:}

It is a very good sign that most of our physicians were found vaccinated and no one was found seropositive. Moreover, who did not take vaccination, all of them reassured that they would complete the vaccination schedule as early as possible. $75 \%$ physicians considered HBV vaccine expensive. However, all of the physicians who participated in this study strongly agreed that HBV Vaccination should be compulsory for every physician.

\section{Recommendations:}

HBV Vaccination should be compulsory for every physician. Proper vaccination schedule should be completed. Checking of antibody titre after completion of vaccination schedule, should be done to find whether the physician is protected or not. Physicians should practice of using protective clothings and equipments during any procedure. They should be more cautious during any invasive procedure to prevent needle stick injury. Young physicians (like Intern doctors) should be vaccinated before their clinical career.

\section{References}

1. Center for Disease Control (CDC). A comprehensive immunization strategy to eliminate transmission of hepatitis B virus infection in the United States. Morbidity and Mortality Weekly Report 2006; 55(RR16): 1-25.

2. Koff R. Immunologically mediated extrahepatic manifestations of viral hepatitis. In: Krawitt EL, Wiesner RH, eds. Autoimmune Liver Disease. New York: Raven press; 1991.

3. WHO. The Global Alliance for Vaccines and Immunization. Hepatitis B Fact Sheet 2008. Available at (http://www.who.int/entity/ immunization delivery/adc/gavi.hepb.facts heet.pdf) [Accessed July 23, 2008].

4. South African Vaccination and Immunisation Centre (SAVIC).Clinical disease hepatitis B. 2008. 
Available at (http://www.savic.ac.za/ disease.php? sub3=88). [Accessed July 17, 2008].

5. Breining Institute: Hepatitis B Fact Sheet 2008. Available at (www.addictionspecialists.com/pdf/ ce1311P2.pdf) [Accessed July 21, 2008].

6. Davis LG, Weber DJ, Lemon SM. Horizontal transmission of hepatitis B virus. Lancet 1989; 1(8643): 889-93.

7. Hollinger FB, Liang TJ. Hepatitis B Virus. In: Knipe DM, et al., eds. Fields Virology. $4^{\text {th }}$ ed. Philadelphia: Lippincott Williams \& Wilkins; 2001

8. Kermode M, Jolley D, Langkham B, Thomas MS, Crofts N. Occupational exposure to blood and risk of blood borne virus infection among health care workers in rural north Indian health care settings. Am J Infect Control 2005; 33: 34-41.

9. Suckling RM, Taegtmeyer M, Nguku PM, AlAbri SS, Kibaru J, Chakaya JM, et al. Susceptibility of health care workers in Kenya to hepatitis B: new strategies for facilitating vaccination uptake. J Hosp Infect 2006; 64(3): 271-7.

10. De Villiers HC, Nel M, Prinsloo EAM. Occupational exposure to bloodborne viruses amongst medical practitioners in Bloemfontein, South Africa. SA Fam Pract 2007; 49(3): 14

11. Mahtab MA, Rahman S, Karim MF, Khan M, Foster G, Solaiman S, et al. Epidemiology of hepatitis B virus in Bangladeshi general population. Hepatobiliary Pancreat Dis Int 2008; 7(6): 595600.

12. McGrane J, Staines A. Nursing staff knowledge of the hepatitis B virus including attitudes and acceptance of hepatitis B vaccination: development of an effective program. AAOHN J 2003; 51(8): 347-52

13. Kane M, Banatvala J, Da Villa G, Esteban R, Franco E, Goudeau A, et al. Consensus statement: Are booster immunizations needed for lifelong hepatitis B immunity? European Consensus Group on Hepatitis B Immunity. Lancet 2000; 355(9203): 561-5

14. Shepard CW, Finelli SL, Fiore AE, Bell BP. Hepatitis B Virus Infection: epidemiology and vaccination. Epidemiol Rev 2006; 28: 112-25.

15. Stein AD, Makarawo TP, Ahmad MFR. A survey of doctors' and nurses' knowledge, attitudes and compliance with infection control guidelines in Birmingham teaching hospitals. J Hosp Infect 2003; 54: 68-73. 